\title{
Seed Plumpness of Rice with Inhibition Expression of Starch Branching Enzymes and Starch Properties, Grain Position on Panicle
}

\author{
Juan Wang ${ }^{1,2}$, Zichun Chen ${ }^{1,2}$, Pan Hu ${ }^{1,2}$, Lingxiao Zhao ${ }^{1,2}$, Lingshang Lin ${ }^{1,2}$ and \\ Cunxu Wei $1,2, *$ (D) \\ 1 Key Laboratory of Crop Genetics and Physiology of Jiangsu Province/Key Laboratory of Plant Functional \\ Genomics of the Ministry of Education, Yangzhou University, Yangzhou 225009, China; \\ juanwang@yzu.edu.cn (J.W.); mx120170762@yzu.edu.cn (Z.C.); m150779@yzu.edu.cn (P.H.); \\ d15016@yzu.edu.cn (L.Z.); 18252713442@163.com (L.L.) \\ 2 Co-Innovation Center for Modern Production Technology of Grain Crops of Jiangsu Province/Joint \\ International Research Laboratory of Agriculture \& Agri-Product Safety of the Ministry of Education, \\ Yangzhou University, Yangzhou 225009, China \\ * Correspondence: cxwei@yzu.edu.cn; Tel.: +86-514-8799-7217; Fax: +86-514-8797-1747
}

Received: 30 July 2018; Accepted: 1 November 2018; Published: 4 November 2018

\begin{abstract}
Cereal crops with inhibition expression of starch branching enzyme (SBE) contain highly resistant starch in the endosperm, and have potential health benefits for human. However, seed plumpness is significantly different, with different inhibition effects of SBE expression, resulting in differently shrunken seeds. In this study, a transgenic resistant starch rice line, which has highly resistant starch and is developed through inhibition expression of SBEs, had non-, slightly, and moderately shrunken seeds with plumpness from high to low. The differently shrunken seeds had significantly different seed weight and starch content. Different morphological starch granules were detected in the endosperm and had similar spatial distribution pattern among the non-, slightly, and moderately shrunken seeds. Starches from differently shrunken seeds had similar amylose content and amylopectin structure. The protein amount of amylose/amylopectin synthesis key enzymes in endosperm was no different between differently shrunken seeds. The primary branch of the panicle had a higher percentage of non-shrunken seeds than did the secondary branch at the same part of the panicle. From the upper part to the base of the panicle, non-shrunken seeds gradually decreased but slightly and moderately shrunken seeds gradually increased. The above results indicated that the differently shrunken seeds in transgenic rice line had the same SBE dosage, and the starch morphology and structure had no relationship with seed plumpness. The grain position on the panicle influenced seed plumpness, but had no effect on starch properties.
\end{abstract}

Keywords: rice; inhibition expression of starch branching enzyme; seed plumpness; grain position on panicle; starch morphology; starch structure

\section{Introduction}

Starch is the main component of cereal endosperm. Its content and properties determine the quality and utilization of cereal crops. Starch contains rapidly digestible starch, slowly digestible starch, and resistant starch (RS) according to its digestion rate. RS is a portion of starch that cannot be degraded in the upper gastrointestinal tract but functions as a substrate for bacterial fermentation in the large intestine [1]. RS can provide many health benefits for humans. For example, RS-enriched food can lower the glycemic and insulin responses and reduce the risk of developing obesity, cardiovascular diseases, and type II diabetes [2-4]. However, normal cereal crops contain very low RS in endosperm. Therefore, it is of interest and importance to cultivate high-RS cereal crops for breeders [3-6]. 
Starch consists of two main components: linear amylose and highly branched amylopectin. In the cereal endosperm, granule-bound starch synthase I (GBSSI) is responsible for amylose synthesis, and starch synthase (SS), starch branching enzyme (SBE), and starch debranching enzyme (DBE) cooperatively synthesize the amylopectin [7]. In addition, starch phosphorylase 1 (Pho1) also plays a role in starch synthesis [8]. The inhibition expression of SBE in cereal endosperm can change amylopectin structure and increase amylose content, leading to the improvement of RS [3-6,9-12]. In cereal endosperm, SBE has three isoforms, SBEI, SBEIIa, and SBEIIb. The SBEIIb mutation, also designated as an amylose extender (ae) mutant, can lead to amylose increase of $10 \%$ and $30 \%$ in rice and maize, respectively [5,9]. The inhibition expression of SBEIIa produces $55.7 \%$ and $38 \%$ amylose content in wheat and barley, respectively [3,12]. A higher increase of amylose content is detected when two or three SBEs inactivation are combined. For example, simultaneous inhibition of SBEIIa and SBEIIb improves amylose content up to $76.2 \%$ in barley [10], while the concerted repression of SBEI, SBEIIa, and SBEIIb can develop an amylose-only starch in barley [6]. Though the above crops with inhibition expression of SBE have high amylose, the amylopectin content significantly decreases and its branch-chains markedly elongate $[5,6,9,10,12]$. Their endosperm is rich in RS and can improve health indices in normal and diabetic rats $[3,4]$.

In cereal crops with inhibition expression of SBE, seed plumpness and weight decrease due to reduced starch synthesis in endosperm. For example, in the rice ae mutant, starch content drops by $50 \%[9,13]$. A similar phenotype is also detected in the maize ae mutant [14]. Furthermore, along with the addition of SBEI mutation in maize ae background, the shrunken seed phenotype is more evident than that of the SBEIIb mutation alone [14]. In SBEIIa ${ }^{-} / \mathrm{SBEIIb}^{-}$transgenic barley, the wrinkled seed is also detected and starch content decreases to $45.4 \%$ from $60 \%$ [10]. Amylose-only barley seeds show $47.2 \%$ starch content, which is slightly lower than that of the control (52.8\%) [6].

A transgenic resistant starch rice line (TRS) has been developed through antisense RNA inhibition of SBEI/SBEIIa/SBEIIb in the background of an indica cultivar Te-qing (TQ) $[4,15]$. TRS seeds have four differently morphological starch granules, including polygonal, aggregated, elongated, and hollow granules, that are regionally distributed in a single seed from inside to outside of the endosperm [16,17]. The TRS endosperm contains abundant RS and has shown significant potential to improve the health of the large bowel in rats [4]. The amylose content in TRS seed is approximately $40 \%$ higher than that of TQ, but the amylopectin content per seed in TRS decreases by approximately 70\%, leading to markedly reduced starch content and seed weight [15]. TRS seeds, except for seriously shrunken seeds, can be classified into non-, slightly, and moderately shrunken seeds according to the plumpness of the seed. The differently shrunken seeds come from the same panicle. However, it is unclear whether the starch morphology and structure are different among differently shrunken seeds. To our knowledge, no papers have reported the reason for these differently shrunken seeds in the same mutant or transgenic line with inhibition expression of SBE.

In this study, seed characteristics, starch morphology and structure, protein amount of key amylose/amylopectin synthesis enzymes, and grain position on panicle were investigated in differently shrunken seeds of TRS through morphological observation, physicochemical analysis, and Western blot methods. Our objective was to reveal the starch properties and discover the reason for the differently shrunken seeds in TRS. This study would provide important information for quality breeding and seed utilization of cereal crops through inhibition expression of SBE.

\section{Materials and Methods}

\subsection{Plant Materials}

The transgenic resistant starch rice line (TRS) was developed through antisense RNA inhibition of SBEI, SBEIIa, and SBEIIb in the background of an indica rice cultivar Te-qing (TQ) $[4,15]$. The expression levels of SBEI, SBEIIa, and SBEIIb in TRS are about $8.5 \%, 10.0 \%$, and $10.7 \%$, respectively, of those in TQ [15]. TRS is a stable transgenic line chosen from four independent homozygous transgenic 
lines [4], and has been planted for 15 generations. TRS was cultivated in a closed transgenic experiment field of Yangzhou University, Yangzhou, China. Mature panicles from the single transgenic line TRS were used as plant materials. Grains were dehulled by hands, and the resulting seeds were used to investigate seed characteristics, starch morphology and structure, and the protein amount of key amylose/amylopectin synthesis enzymes.

\subsection{Determination of Seed Weight and Its Starch and Soluble Sugar Contents}

Dry seeds were weighed to calculate the seed weight. The starch and soluble sugar contents in seed were measured following the method of Gao et al. [18]. Briefly, dry seeds were extensively ground with a mortar and passed through a 100-mesh sieve to obtain seed flour. The soluble sugar in seed flour was extracted using $80 \%(v / v)$ ethanol three times, and the starch in flour precipitate was hydrolyzed to soluble sugar using $\mathrm{HClO}_{4}$. Finally, the soluble sugar content was determined using anthrone- $\mathrm{H}_{2} \mathrm{SO}_{4}$ method and converted to soluble sugar and starch contents.

\subsection{Seed Sections for Light Microscopy}

The whole sections of mature seeds were prepared exactly following the method of Zhao et al. [19]. The semithin sections with $2 \mu \mathrm{m}$ thickness were sectioned under an ultrathin microtome (EM UC7), stained with $\mathrm{I}_{2} / \mathrm{KI}$, and observed using an Olympus BX53 light microscope equipped with a CCD camera.

\subsection{Molecular Weight Distribution of Starch}

Total starch was isolated from seeds following the method of Man et al. [20]. The debranched starch was prepared exactly by following the method of Lin et al. [21]. Briefly, isolated starch was deproteinized with protease and sodium bisulfite, dissolved in DMSO solution, and precipitated with $80 \%$ ethanol. The precipitated starch was dispersed in water and debranched using isoamylase. The debranched starch was analyzed using a gel-permeation chromatography (GPC) system (PL-GPC 220) with three columns (PL110-6100, 6300, 6526) and a differential refractive index detector, as described in the method of Man et al. [20].

\subsection{Western Blot}

The above isolated starches with the same weight were dispersed in a gelatinized buffer (50 mM Tris- $\mathrm{HCl}, \mathrm{pH} 8.0,10 \% \mathrm{SDS}$ ) and boiled in a water bath with constant stirring to release the granule-bound protein. After centrifuging, the soluble fraction was precipitated in acetone overnight to obtain the protein pellet. The protein pellet was suspended in the same volume of gelatinized buffer. The same volume of protein suspension was loaded to the SDS gels and then electrophoretically transferred from SDS gels to polyvinylidene difluoride membranes. These blots were soaked in TBST buffer ( $50 \mathrm{mM}$ Tris-HCl, pH 7.4, 0.1\% Tween 20, $0.2 \mathrm{M} \mathrm{NaCl}$ ) containing $5 \%$ milk to block non-specific sites and then exposed to antibodies at $4{ }^{\circ} \mathrm{C}$ overnight. After washing, the blots were probed by goat anti-rabbit immunoglobulins conjugated to horseradish peroxidase. Detection was conducted using ECL detection reagents, and imaging was detected by a chemiluminescence analyzer. Antibodies against SBEI, SBEIIa, SBEIIb, Pho1, SSI, and GBSSI were described previously [22].

\subsection{Analysis of Grain Position on Panicle}

The growing position on panicle of differently shrunken seeds was analyzed according to the method described by Dong et al. [23] with some modifications. All branches of the panicle were classified into three parts: the upper, middle, and basal (Figure 1). The growing positions of grains were numbered 1 to 6 on the primary branch from the top to the bottom and 1 to 3 or 4 on the secondary branch. Ten random panicles from different lines were chosen as one replication, and three replications were conducted. 


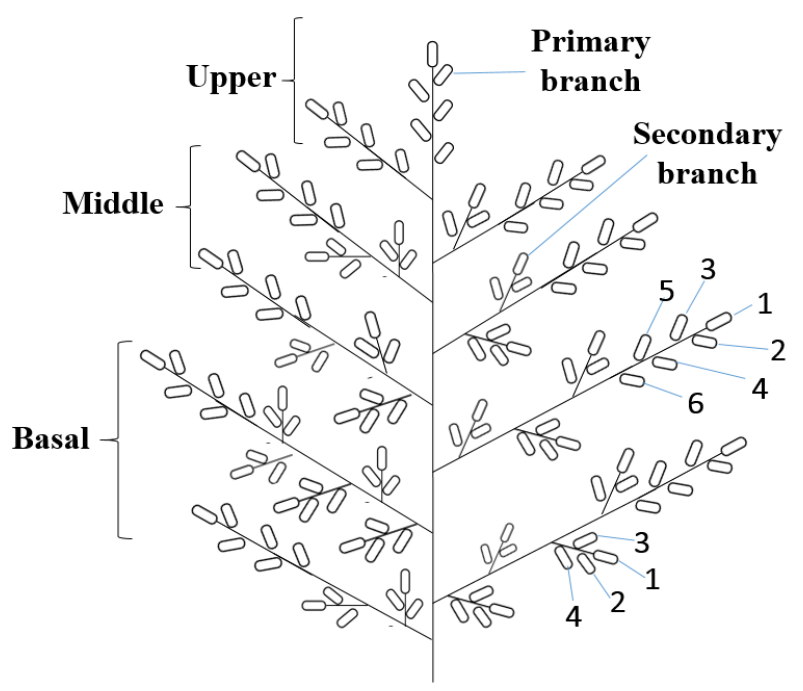

Figure 1. Diagram of rice panicle and grain growing position.

\subsection{Statistical Analysis}

The data were analyzed using SPSS 16.0 Statistical Software Program. The one-way analysis of variance was evaluated using Tukey's test.

\section{Results}

\subsection{Characteristics of Differently Shrunken Seeds}

TRS mature seeds showed an opaque phenotype. These seeds, except for the seriously shrunken ones, were classified into three types, non-, slightly, and moderately shrunken seeds, according to their plumpness and morphology (Figure 2). The seriously shrunken seeds might be due to the endosperm development having stopped at the middle stage of kernel development. This phenomenon also exists in normal rice. Therefore, they were ignored in the present study. The seed testing of three successive years showed that $18.9 \sim 19.6 \%$ of seeds had a non-shrunken phenotype, 37.2 39.1\% of seeds were slightly shrunken, and $41.3 \sim 43.9 \%$ of seeds were moderately shrunken.

The seed weight, starch content, and soluble sugar content in non-, slightly, and moderately shrunken seeds are shown in Figure 3. It was clear that seed weight and starch content significantly decreased from non-shrunken to slightly shrunken and moderately shrunken seeds. However, the soluble sugar content in moderately shrunken seed was significantly higher than in non- and slightly shrunken seeds.
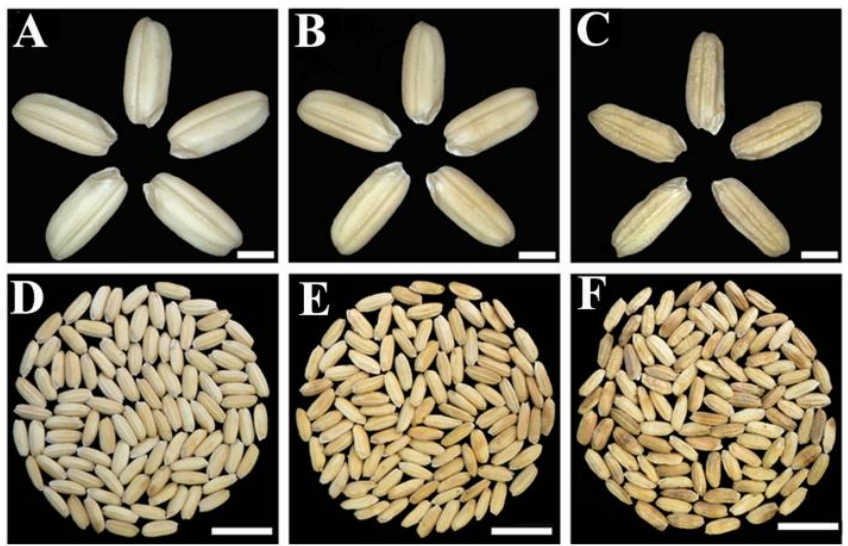

Figure 2. Morphology of non-shrunken (A,D), slightly shrunken (B,E), and moderately shrunken seeds $(\mathbf{C}, \mathbf{F})$. Scale bar $=2 \mathrm{~mm}$ for $(\mathbf{A}-\mathbf{C})$ and $5 \mathrm{~mm}$ for $(\mathbf{D}-\mathbf{F})$. 


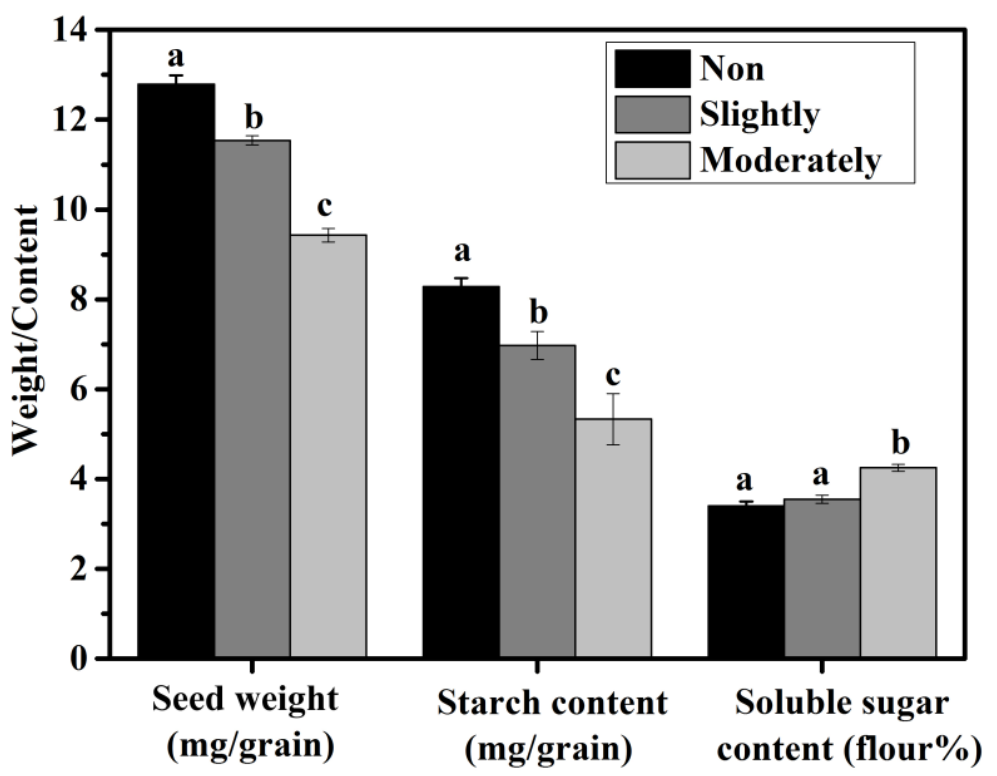

Figure 3. Seed weight, starch content, and soluble sugar content of differently shrunken seeds. Data are means \pm SD from three biological replicates. Values for the same seed characteristic with different letters are significantly different $(p<0.05)$.

\subsection{Starch Morphology in Differently Shrunken Seeds}

Our previous study showed that TQ seed had homogeneous compound starch granules in endosperm, whereas TRS endosperm contained differently morphological starches including polygonal, aggregated, elongated, and hollow granules $[16,17]$. To investigate whether the morphology of starch granules in non-, slightly, and moderately shrunken seeds was different from each other, the semithin section of the whole seed was stained with $\mathrm{I}_{2} / \mathrm{KI}$ (Figure 4). The polygonal, aggregated, elongated, and hollow starch granules were all observed from the inside to the outside of the endosperm in the non-, slightly, and moderately shrunken seeds. The innermost polygonal granule contained subgranules of compound starch, as in normal rice (Figure 4(A1,B1,C1)). The aggregated starch granule was a compound starch but circled by a thick band (Figure 4(A2,B2,C2)). When separating starches from TRS endosperm, the aggregated starch granule can maintain its complete morphology, while a compound starch is disassociated into several polygonal subgranules [16]. The elongated starch granule consisted of two or three polygonal starch granules in line to form an elongated morphology (Figure 4(A3,B3,C3)) [16]. The outermost hollow starch granule was hollow inside and only contained a thick starch band (Figure 4(A4,B4,C4)) [16]. The morphology and distribution of the abovementioned polygonal, aggregated, elongated, and hollow starch granules showed no significant differences in non-, slightly, and moderately shrunken seeds. However, the packed degree of these starch granules was significantly different among differently shrunken seeds. The starch granules in the non-shrunken seed were arranged more tightly than in the slightly shrunken seed, followed by the moderately shrunken seed (Figure 4). The present results indicated that the shrunken phenotype was related to the packed degree of starch granules, and the more densely packed the starch granules with high plumpness, the less shrunken the seed. 


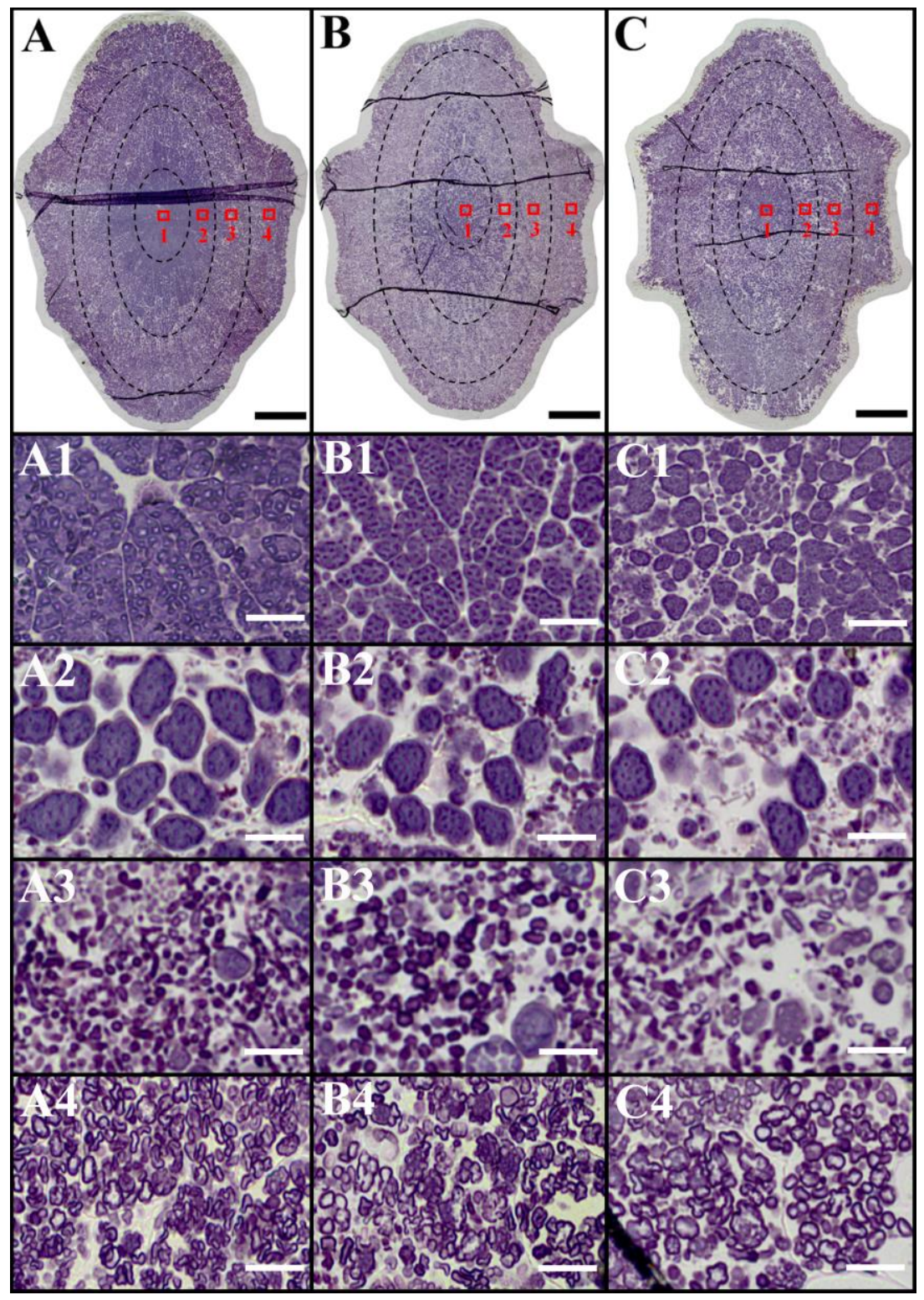

Figure 4. Morphology of starch granules in non-shrunken (A), slightly shrunken (B), and moderately shrunken seeds (C). The whole seed was transversely sectioned at the mid-region of the seed. (A1-A4), (B1-B4), and (C1-C4) are magnified regions responsible for the square areas in (A), (B), and (C), respectively. Scale bar $=300 \mu \mathrm{m}$ for $(\mathbf{A}-\mathbf{C})$ and $20 \mu \mathrm{m}$ for (A1-C4).

\subsection{Molecular Weight Distribution of Starch from Differently Shrunken Seeds}

To investigate whether starches from differently shrunken seeds had different starch structures, total starch was isolated from mature seed. The molecular weight distribution of isoamylase-debranched starch as measured by GPC is shown in Figure 5. A trimodal distribution composed of Peak 1, Peak 2, and Peak 3 was identified. Peak 1 and Peak 2 represent short and long branch-chains of amylopectin, respectively, and Peak 3 consists mainly of amylose [24]. The starches from differently shrunken seeds all displayed similar molecular weight distributions (Figure 5). The contents of amylopectin short-branch chain, amylopectin long chain, and amylose, and the ratio of short and long branch-chain of amylopectin showed no differences among differently shrunken seeds (Table 1), indicating that non-, slightly, and moderately shrunken seeds had a similar starch structure. 


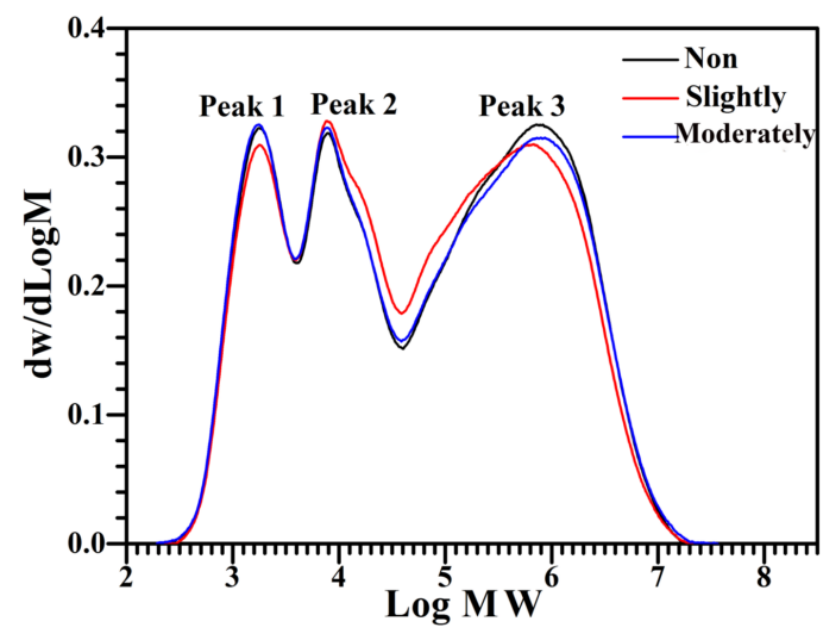

Figure 5. GPC profiles of isoamylase-debranched starches from non-, slightly, and moderately shrunken seeds.

Table 1. GPC parameters of isoamylase-debranched starches.

\begin{tabular}{ccccc}
\hline & \multicolumn{3}{c}{ Peak Area } & \multirow{2}{*}{ Area Ratio of Peak 1 to Peak 2 } \\
\cline { 2 - 4 } & Peak 1 (\%) & Peak 2 (\%) & Peak 3 (\%) & \\
\hline Non-shrunken seed & $20.9 \pm 0.2 \mathrm{a}$ & $23.8 \pm 0.8 \mathrm{a}$ & $55.3 \pm 0.6 \mathrm{a}$ & $0.88 \pm 0.04 \mathrm{a}$ \\
Slightly shrunken seed & $20.9 \pm 1.8 \mathrm{a}$ & $24.9 \pm 1.7 \mathrm{a}$ & $54.1 \pm 0.2 \mathrm{a}$ & $0.84 \pm 0.13 \mathrm{a}$ \\
Moderately shrunken seed & $21.4 \pm 0.9 \mathrm{a}$ & $24.7 \pm 0.1 \mathrm{a}$ & $53.9 \pm 0.8 \mathrm{a}$ & $0.87 \pm 0.04 \mathrm{a}$ \\
\hline
\end{tabular}

Data are means $\pm \mathrm{SD}, n=3$. Values in the same column with same letters are not significantly different $(p>0.05)$.

\subsection{Protein Amount of Amylose/Amylopectin Synthesis Key Enzymes in Differently Shrunken Seeds}

GBSS, SS, SBE, and Pho1 are key enzymes responsible for the synthesis of amylose and amylopectin [7]. The composition of starch granule-bound proteins is thought to reflect the composition of the starch biosynthetic protein complex $[25,26]$. Though SBEI in wild-type rice is detected in only soluble protein and loosely bound protein, it and soluble starch synthases can be trapped in the starch granules in the absence of SBEIIb protein [27]. To investigate whether the different starch contents in differently shrunken seeds were caused by different dosages of GBSS, SS, SBE, and Pho1, Western blotting against SBEI, SBEIIa, SBEIlb, Pho1, SSI, and GBSSI was conducted. Results showed that no significant difference was detected in the protein amounts of SBEI, SBEIIa, SBEIIb, Pho1, SSI, and GBSSI in isolated starches from non-, slightly, and moderately shrunken seeds (Figure 6), indicating that the amounts of these enzymes were not significantly different among differently shrunken seeds.

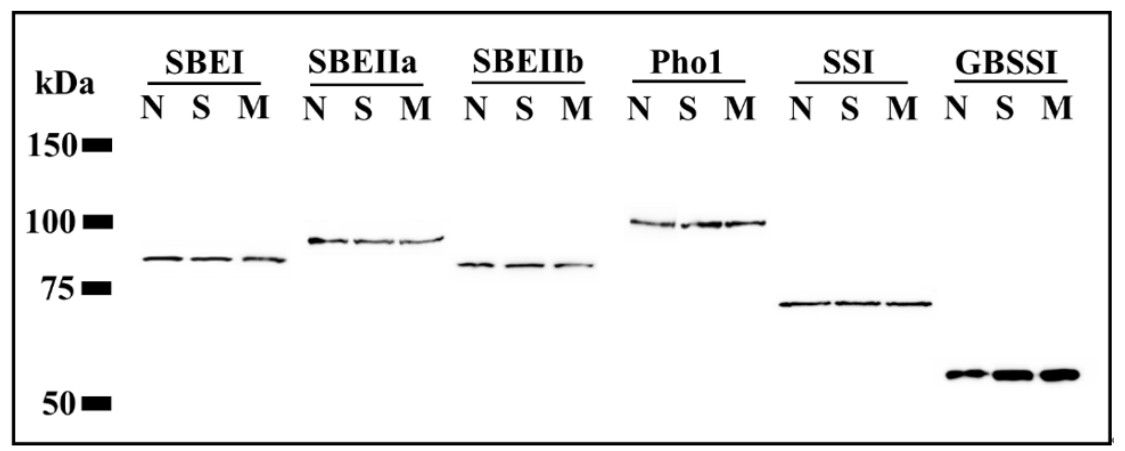

Figure 6. Protein amounts of amylose/amylopectin synthesis key enzymes in starches from non-shrunken (N), slightly shrunken (S), and moderately shrunken seeds (M). The protein amount is standardized by milligrams of starch. 


\subsection{Growing Position on Panicle of Differently Shrunken Seeds}

It has been widely reported that seed development differs in different grain positions on the rice panicle. Generally, grains situated the upper primary branches of the panicle flower earlier, fill more rapidly, and achieve a bigger and heavier seed than grains situated on the lower secondary branches [28-31]. Therefore, to detect whether there was a position effect of panicle on differently shrunken seeds, the distributions of non-, slightly, and moderately shrunken seeds on the panicle were investigated. As shown in Figure 7, the upper primary branches had the highest ratio of non-shrunken seeds but the lowest ratio of moderately shrunken seeds. From the upper to the basal, non-shrunken seeds in the primary branch gradually decreased, but slightly and moderately shrunken seeds gradually increased. In basal primary branches, there were extremely low non-shrunken seeds but relatively high slightly and moderately shrunken seeds. For secondary branches, they normally contained few non-shrunken seeds. Even in the upper secondary branch, the highest ratio of non-shrunken seeds was not more than $40 \%$. From the upper to the basal, slightly shrunken seeds on secondary branches gradually decreased while moderately shrunken seeds increased. Compared with primary branches, secondary branches on the same part of the panicle generally had a lower percentage of non-shrunken seeds but a higher percentage of moderately shrunken seeds. The above results indicate that the grains located on primary branches developed better than those on secondary branches, and the upper ones developed better than those of the middle, followed by the basal. Overall, differently shrunken seeds were spatially distributed on the panicle and there was an obvious position effect of panicle on seed plumpness.
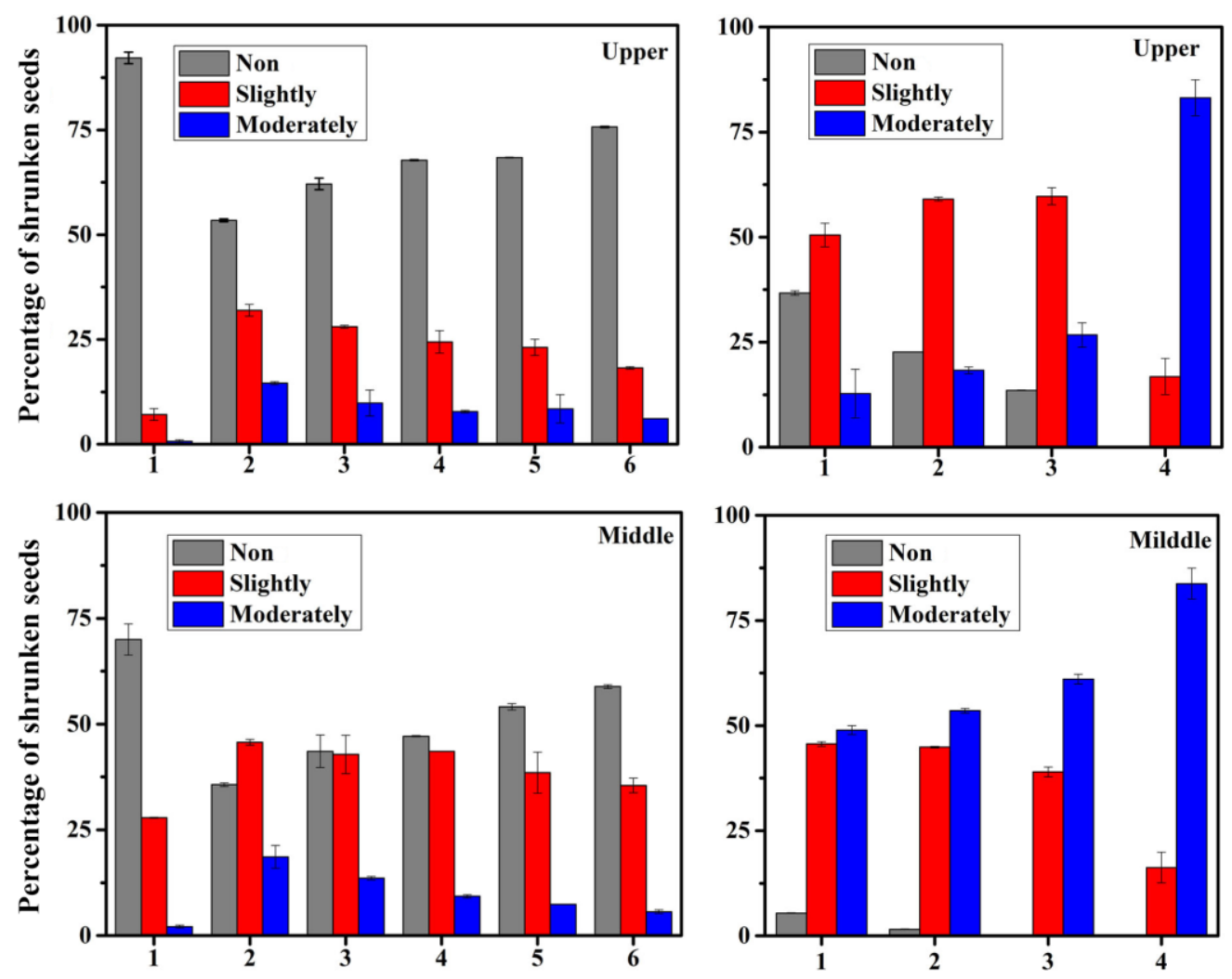

Figure 7. Cont. 

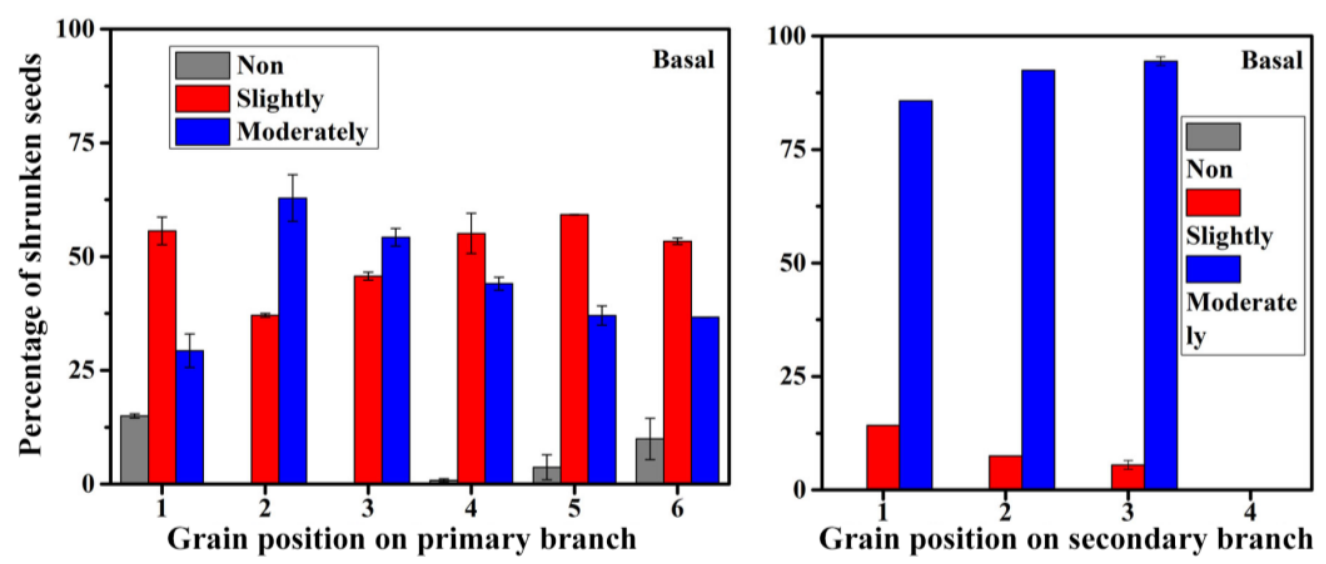

Figure 7. Distributions of differently shrunken seeds on rice panicle. The number of the grain position on the panicle is shown in Figure 1. Data are means \pm SD from three biological replicates.

\section{Discussion}

\subsection{Morphology and Structure of Starch and Seed Plumpness}

The inhibition expression of SBE in endosperm can change amylopectin structure and amylose content, form different morphological starch granules, and increase RS content in endosperm [3-6,9-12]. The above variation ranges are significantly related to the dosage of SBE. Therefore, many cereal crops with inhibition of SBE expression have been cultivated to provide the starches with different nutritional qualities [3,4,11,32]. For example, a series of rice transgenic lines with different SBEIlb contents are cultivated through introducing SBEIIb to the ae mutant [32]. The starch granule morphology in these transgenic lines is variable, and the amylopectin structure is significantly influenced by the SBEIlb dosage. The SBE dosage is positively correlated with short branch-chains of amylopectin but negatively correlated with long branch-chains. Two transgenic rice lines with downregulating SBEIIb expression are cultivated through artificial microRNA- (amiRNA) and Hairpin RNA-mediated (hp-RNA) RNA silencing [11]. The expression of SBEIIb decreases more pronouncedly in the amiRNA line than in the hp-RNA line, leading to a greater increase in long and intermediate branch-chains of amylopectin and more severe alterations in starch morphology in the amiRNA line than in the hp-RNA line. In this study, non-, slightly, and moderately shrunken seeds were identified in rice TRS with inhibition of SBEI/SBEIIa/SBEIIb expression (Figure 2). Differently shrunken seeds had a significantly different seed weight and starch content (Figure 3). Though different morphological starch granules were detected in endosperm, their distributions had no difference among differently shrunken seeds (Figure 4). The molecular weight distribution of starch indicated that starch structure, including amylose content and amylopectin short and long branch-chains, was similar among differently shrunken seeds (Figure 5). The protein amounts of GBSSI, SBEI, SBEIIa, SBEIlb, Pho1, and SSI in endosperm were also similar among differently shrunken seeds (Figure 6), and agreed with their starch structure. Though the differently shrunken seeds from different transgenic lines with inhibition of SBE expression have different starch morphology and structure [11,32], the present results indicated that starch morphology and structure had no relationship with seed plumpness. The seeming contradiction might be due to the different transgenic lines having different dosages of SBE but the differently shrunken seeds in the same transgenic line had the same SBE dosage.

\subsection{Grain Position on Panicle and Seed Plumpness}

In panicles of normal rice cultivars, grains situated on the upper primary branch and the basal secondary branch have the highest and lowest kernel filling rate and exhibit the heaviest and lightest seed weight, respectively. Therefore, the former and the latter grains are named the superior and inferior grain, respectively $[23,29,30]$. In the present study, differently shrunken seeds were related 
to their growing positions on the panicle. The grains on the primary branch developed better than those on the secondary branch, and the grains on the upper branch developed better than those on the middle branch, followed by the basal branch (Figure 7). This phenomenon was similar to that of superior/inferior grain. The mechanism leading to the superior/inferior grain in rice has been investigated in some studies on starch-rich endosperm as a sink drawing photo assimilate towards it. The superior grains have a higher cell division and kernel-filling rate in endosperm than the inferior grains [29]. A series of starch metabolism-related genes show higher transcript levels in superior grains than in inferior grains at the early and middle kernel-filling stages [30]. In addition, the photo assimilate supply is also considered an important factor in the poor filling of inferior grain. The kernel-filling of inferior grain significantly increases after removal of superior grain due to the assimilate, which should be drawn towards the superior grain, being transported to the inferior grain [33,34]. In the present study, a higher rate of starch synthesis and a lower rate of grain filling were detected in the grains on the upper and basal branches of the panicle, respectively, although the three SBEs were inhibited in them. This also indicated that the assimilate supply was a significant factor in grain development.

The inferior grain is also related to the decrease in starch synthesis and the accumulation of soluble sugar in the developing kernel. It has been reported that the inferior grain has a higher concentration of sucrose than the superior grain [28]. The high concentration of sucrose in inferior grain is attributed to the low activity of sucrose synthase in the developing kernel, which catalyzes and degrades sucrose to produce uridine diphosphoglucose and fructose [34,35]. Yang et al. [29] detected that the changes in soluble sugar are closely related to those of sucrose in the spikelet, indicating that the high concentration of soluble carbohydrate in inferior grains is mainly due to the high concentration of sucrose in the inferior spikelet. In the present study, the soluble sugar in the three shrunken seeds of TRS showed a gradually increasing trend with the decrease in seed plumpness (Figure 3). It could be that the gradual increase in sucrose accumulation resulted from the gradually decreased activity of sucrose synthase.

\section{Conclusions}

Rice TRS with inhibition expression of SBEs had non-, slightly, and moderately shrunken seeds due to different seed plumpness. Seed weight, starch content, and soluble sugar content were significantly different among differently shrunken seeds. However, starch morphology, starch structure, and amylose/amylopectin synthesis key enzymes were similar among non-, slightly, and moderately shrunken seeds, indicating that seed plumpness had no effect on starch properties. The grain growing position on the panicle affected the plumpness of seeds, resulting in differently shrunken seeds.

Author Contributions: J.W. and C.W. conceived the study and designed the experiments. J.W., Z.C., P.H., L.Z. and L.L. performed the experiments. J.W. and C.W. analyzed the data and wrote the manuscript. All authors discussed the contents of the manuscript and approved the submission.

Funding: This research was supported by the Natural Science Foundation of Jiangsu Province (BK20160457), the China Postdoctoral Science Foundation (2016590509), the Qing Lan Project of Jiangsu Province, the Talent Project of Yangzhou University, and the Priority Academic Program Development of Jiangsu Higher Education Institutions.

Acknowledgments: The authors are grateful to Xiuling Cai (Institute of Plant Physiology \& Ecology, Chinese Academy of Sciences) and Xiangbai Dong (Institute of Botany, Chinese Academy of Sciences) for kindly providing the antibodies of starch synthesis related enzymes.

Conflicts of Interest: The authors declare no conflict of interest.

\section{References}

1. Englyst, H.N.; Kingman, S.M.; Cummings, J.H. Classification and measurement of nutritionally important starch fractions. Eur. J. Clin. Nutr. 1992, 45, 33-50.

2. Nugent, A.P. Health properties of resistant starch. Nutr. Bull. 2005, 30, 27-54. [CrossRef] 
3. Regina, A.; Bird, A.; Topping, D.; Bowden, S.; Freeman, J.; Barsby, T.; Kosar-Hashemi, B.; Li, Z.; Rahman, S.; Morell, M. High-amylose wheat generated by RNA interference improves indices of large-bowel health in rats. Proc. Natl. Acad. Sci. USA 2006, 10, 3546-3551. [CrossRef] [PubMed]

4. Zhu, L.; Gu, M.; Meng, X.; Cheung, S.C.K.; Yu, H.; Huang, J.; Sun, Y.; Shi, Y.; Liu, Q. High-amylose rice improves indices of animal health in normal and diabetic rats. Plant Biotech. J. 2012, 10, 353-362. [CrossRef] [PubMed]

5. Li, L.; Jiang, H.; Campbell, M.; Blanco, M.; Jane, J.L. Characterization of maize amylose-extender (ae) mutant starches. Part I: relationship between resistant starch contents and molecular structures. Carbohyd. Polym. 2008, 74, 396-404. [CrossRef]

6. Carciofi, M.; Blennow, A.; Jensen, S.L.; Shaik, S.S.; Henriksen, A.; Buléon, A.; Holm, P.B.; Hebelstrup, K.H. Concerted suppression of all starch branching enzyme genes in barley produces amylose-only starch granules. BMC Plant Biol. 2012, 12, 223. [CrossRef] [PubMed]

7. Tian, T.; Qian, Q.; Liu, Q.; Yan, M.; Liu, X.; Yan, C.; Liu, G.; Gao, Z.; Tang, S.; Zeng, D.; Wang, Y.; Yu, J.; Gu, M.; $\mathrm{Li}, \mathrm{J}$. Allelic diversities in rice starch biosynthesis lead to a diverse array of rice eating and cooking qualities. Proc. Natl. Acad. Sci. USA 2009, 106, 21760-21765. [CrossRef] [PubMed]

8. Satoh, H.; Shibahara, K.; Tokunaga, T.; Nishi, A.; Tasaki, M.; Hwang, S.K.; Okita, T.W.; Kaneko, N.; Fujita, N.; Yoshida, M. Mutation of the plastidial $\alpha$-glucan phosphorylase gene in rice affects the synthesis and structure of starch in the endosperm. Plant Cell 2008, 20, 1833-1849. [CrossRef] [PubMed]

9. Nishi, A.; Nakamura, Y.; Tanaka, N.; Satoh, H. Biochemical and genetic analysis of the effects of amylose-extender mutation in rice endosperm. Plant Physiol. 2001, 127, 459-472. [CrossRef] [PubMed]

10. Regina, A.; Kosar-Hashemi, B.; Ling, S.; Li, Z.; Rahman, S.; Morell, M. Control of starch branching in barley defined through differential RNAi suppression of starch branching enzyme IIa and Ilb. J. Exp. Bot. 2010, 61, 1469-1482. [CrossRef] [PubMed]

11. Butardo, V.M.; Fitzgerald, M.A.; Bird, A.R.; Gidley, M.J.; Flanagan, B.M.; Larroque, O.; Resurreccion, A.P.; Laidlaw, H.K.C.; Jobling, S.A.; Morell, M.K.; Rahman, S. Impact of down-regulation of starch branching enzyme IIb in rice by artificial microRNA- and hairpin RNA-mediated RNA silencing. J. Exp. Bot. 2011, 62, 4927-4941. [CrossRef] [PubMed]

12. Slade, A.J.; McGuire, C.; Loeffl, D.; Mullenberg, J.; Skinner, W.; Fazio, G.; Holm, A.; Brandt, K.M.; Steine, M.N.; Goodstal, J.F.; Knauf, V.C. Development of high amylose wheat through TILLING. BMC Plant Biol. 2012, 12, 69. [CrossRef] [PubMed]

13. Asai, H.; Abe, N.; Matsushima, R.; Crofts, N.; Oitome, NF.; Nakamura, Y.; Fujita, N. Deficiencies in both starch synthase IIIa and branching enzyme IIb lead to a significant increase in amylose in SSIIa inactive japonica rice seeds. J. Exp. Bot. 2014, 65, 5497-5507. [CrossRef] [PubMed]

14. Yao, Y.; Thompson, D.B.; Guiltinan, M.J. Maize starch-branching enzyme isoforms and amylopectin structure. In the absence of starch branching enzyme IIb, the further absence of starch branching enzyme Ia leads to increased branching. Plant Physiol. 2004, 136, 3515-3523. [CrossRef] [PubMed]

15. Wang, J.; Hu, P.; Lin, L.; Chen, Z.; Liu, Q.; Wei, C. Gradually decreasing starch branching enzyme expression is responsible for the formation of heterogeneous starch granules. Plant Physiol. 2018, 176, 582-595. [CrossRef] [PubMed]

16. Wei, C.; Qin, F.; Zhu, L.; Zhou, W.; Chen, Y.; Wang, Y.; Gu, M.; Liu, Q. Microstructure and ultrastructure of high-amylose rice resistant starch granules modified by antisense RNA inhibition of starch branching enzyme. J. Agric. Food Chem. 2010, 58, 1224-1232. [CrossRef] [PubMed]

17. Cai, C.; Huang, J.; Zhao, L.; Liu, Q.; Zhang, C.; Wei, C. Heterogeneous structure and spatial distribution in endosperm of high-amylose rice starch granules with different morphologies. J. Agric. Food Chem. 2014, 62, 10143-10152. [CrossRef] [PubMed]

18. Gao, H.; Cai, J.; Han, W.; Huai, H.; Chen, Y.; Wei, C. Comparison of starches isolated from three different Trapa species. Food Hydrocolloid. 2014, 37, 174-181. [CrossRef]

19. Zhao, L.; Pan, T.; Cai, C.; Wang, J.; Wei, C. Application of whole sections of mature cereal seeds to visualize the morphology of endosperm cell and starch and the distribution of storage protein. J. Cereal Sci. 2016, 71, 19-27. [CrossRef]

20. Man, J.; Lin, L.; Wang, Z.; Wang, Y.; Liu, Q.; Wei, C. Different structures of heterogeneous starch granules from high-amylose rice. J. Agric. Food Chem. 2014, 62, 11254-11263. [CrossRef] [PubMed] 
21. Lin, L.; Cai, C.; Gilber, R.G.; Li, E.; Wang, J.; Wei, C. Relationships between amylopectin molecular structures and functional properties of different-sized fractions of normal and high-amylose maize starches. Food Hydrocolloid. 2016, 52, 359-368. [CrossRef]

22. Liu, D.; Wang, W.; Cai, X. Modulation of amylose content by structure-based modification of OsGBSS1 activity in rice (Oryza sativa L.). Plant Biotech. J. 2014, 12, 1297-1307. [CrossRef] [PubMed]

23. Dong, M.H.; Sang, D.Z.; Wang, P.; Zhang, W.J.; Yang, J.C. Difference in chalky characters of the grains at different positions within a rice panicle. Acta Agron. Sin. 2006, 32, 103-111.

24. Song, Y.; Jane, J. Characterization of barley starches of waxy, normal, and high amylose varieties. Carbohydr. Polym. 2000, 41, 365-377. [CrossRef]

25. Liu, F.; Romanova, N.; Lee, E.A.; Ahmed, R.; Evans, M.; Gilbert, E.P.; Morell, M.K.; Emes, M.J.; Tetlow, I.J. Glucan affinity of starch synthase IIa determines binding of starch synthase I and starch-branching enzyme IIb to starch granules. Biochem. J. 2012, 448, 373-387. [CrossRef] [PubMed]

26. Luo, J.; Ahmed, R.; Kosar-Hashemi, B.; Larroque, O.; Butardo, V.M.; Tanner, G.J.; Colgrave, M.L.; Upadhyaya, N.M.; Tetlow, I.J.; Emes, M.J.; et al. The different effects of starch synthase IIa mutations or variation on endosperm amylose content of barley, wheat and rice are determined by the distribution of starch synthase I and starch branching enzyme IIb between the starch granule and amyloplast stroma. Theor. Appl. Genet. 2015, 128, 1407-1419. [PubMed]

27. Itoh, Y.; Crofts, N.; Abe, M.; Hosaka, Y.; Fujita, N. Characterization of the endosperm starch and the pleiotropic effects of biosynthetic enzymes on their properties in novel mutant rice lines with high resistant starch and amylose content. Plant Sci. 2017, 258, 52-60. [CrossRef] [PubMed]

28. Ishimaru, T.; Hirose, T.; Matsuda, T.; Goto, A.; Takahashi, K.; Sasaki, H.; Terao, T.; Ishii, R.; Ohsugi, R.; Yamagishi, T. Expression patterns of genes encoding carbohydrate- metabolizing enzymes and their relationship to grain filling in rice (Oryza sativa L.): Comparison of caryopses located at different positions in a panicle. Plant Cell Physiol. 2005, 46, 620-628. [CrossRef] [PubMed]

29. Yang, J.; Zhang, J.; Wang, Z.; Liu, K.; Wang, P. Post-anthesis development of inferior and superior spikelets in rice in relation to abscisic acid and ethylene. J. Exp. Bot. 2006, 57, 149-160. [CrossRef] [PubMed]

30. Zhu, G.; Ye, N.; Yang, J.; Peng, X.; Zhang, J. Regulation of expression of starch synthesis genes by ethylene and ABA in relation to the development of rice inferior and superior spikelets. J. Exp. Bot. 2011, 62, 3907-3916. [CrossRef] [PubMed]

31. Das, K.; Panda, B.B.; Sekhar, S.; Kariali, E.; Mohapatra, K.P.; Shaw, P.B. Comparative proteomics of the superior and inferior nspikelets at the early grain filling stage in rice cultivars contrast for panicle compactness and ethylene evolution. J. Plant Physiol. 2016, 202, 65-74. [CrossRef] [PubMed]

32. Tanaka, N.; Fujita, N.; Nishi, A.; Satoh, H.; Hosaka, Y.; Ugaki, M.; Kawasaki, S.; Nakamura, Y. The structure of starch can be manipulated by changing the expression levels of starch branching enzyme IIb in rice endosperm. Plant Biotech. J. 2004, 2, 507-516. [CrossRef] [PubMed]

33. Xu, F.X.; Xiong, H.; Zhu, Y.C.; Wang, G.X. Effect of source-sink ratio on grain filling and the source-sink characteristics of high yield varieties of mid-season hybrid rice. Sci. Agric. Sin. 2005, 38, 265-271.

34. You, C.; Chen, L.; He, H.; Wu, L.; Wang, S.; Ding, Y.; Ma, C. iTRAQ-based proteome profile analysis of superior and inferior spikelets at early grain filling stage in japonica rice. BMC Plant Biol. 2017, 17, 100. [CrossRef] [PubMed]

35. Liang, J.; Zhang, J.; Cao, X. Grain sink strength may be related to the poor grain filling of indica-japonica (Oryza sativa) hybrids. Physiol. Plant. 2001, 112, 470-477. [CrossRef] [PubMed]

(C) 2018 by the authors. Licensee MDPI, Basel, Switzerland. This article is an open access article distributed under the terms and conditions of the Creative Commons Attribution (CC BY) license (http://creativecommons.org/licenses/by/4.0/). 